\title{
A framework for leveraging animal movement to understand spatio-temporal disease dynamics
}

\author{
Mark Wilber ${ }^{1}$, Anni Yang ${ }^{2}$, Raoul Boughton ${ }^{3}$, Kezia Manlove $^{4}$, Ryan Miller ${ }^{5}$, Kim Pepin ${ }^{6}$, \\ and George Wittemyer ${ }^{7}$ \\ ${ }^{1}$ The University of Tennessee Knoxville Institute of Agriculture \\ ${ }^{2}$ USDA-APHIS National Wildlife Research Center \\ ${ }^{3}$ Archbold Biological Station \\ ${ }^{4}$ Utah State University \\ ${ }^{5}$ United States Department of Agriculture, Animal and Plant Health Inspection Service, \\ Veterinary Services, Center for Epidemiology and Animal Health \\ ${ }^{6}$ United States Department of Agriculture Animal and Plant Health Inspection Service \\ ${ }^{7}$ Colorado State University
}

November 17, 2021

\begin{abstract}
The ongoing explosion of fine-resolution movement data in animal systems provides a unique opportunity to empirically quantify spatial, temporal, and individual variation in transmission risk and improve our ability to forecast disease outbreaks. However, we lack a generalizable framework that can leverage movement data to quantify transmission risk and how it affects pathogen invasion and persistence on heterogeneous landscapes. We developed a flexible framework "Movement-driven modeling of spatiotemporal infection risk" (MoveSTIR) that leverages diverse data on animal movement to derive metrics of direct and indirect contact by decomposing transmission into constituent processes of contact formation and duration and pathogen deposition and acquisition. We use MoveSTIR to demonstrate that ignoring fine-scale animal movements on actual landscapes can mischaracterize transmission risk and epidemiological dynamics. MoveSTIR unifies previous work on epidemiological contact networks and can address applied and theoretical questions at the nexus of movement and disease ecology.
\end{abstract}

\section{Hosted file}

direct_indirect_manuscript.pdf available at https://authorea.com/users/441733/articles/ 545842-a-framework-for-leveraging-animal-movement-to-understand-spatio-temporal-diseasedynamics 